\title{
APPLICATION OF THE BOUNDARY ELEMENT METHOD TO THE MICROMECHANICAL ANALYSIS OF COMPOSITE MATERIALS
}

\author{
R. K. Goldberg and D. A. Hopkins \\ NASA Lewis Research Center, Cleveland, OH 44135, U.S.A.
}

\begin{abstract}
A new boundary element formulation for the micromechanical analysis of composite materials is presented in this study. A unique feature of the formulation is the use of circular shape functions to convert the two-dimensional integrations of the composite fibers to one-dimensional integrations. To demonstrate the applicability of the formulations, several example problems including elastic and thermal analyses of laminated composites and elastic analyses of woven composites are presented and the boundary element results compared to experimental observations and/or results obtained through alternate analytical procedures. While several issues remain to be addressed in order to make the methodology more robust, the formulations presented here show the potential in providing an alternative to traditional finite element methods, particularly for complex composite architectures.
\end{abstract}

\section{INTRODLCTION}

In the analysis of composite materials, the material behavior at the micromechanical (constituent) level is often of interest. One major parameter of interest (among several) is the calculation of effective (average) material properties. Several methodologies have been developed previously in order to analyze composite micromechanical behavior for both laminated and woven composite architectures, which are thoroughly reviewed and compared in works such as Refs [1-4]. In the case of unidirectional composite laminates and plain and satin weave woven composites, simplified models have been developed which yield closed form expressions which describe the composite effective properties and certain local parameters. Classical methods discussed in Refs [1-3] for laminated composites include the Voight and Reuss models, the vanishing fiber diameter model, the self-consistent method, the Mori-Tanaka method, the composite spheres model and the method of cells. For plain weave woven composites, Ishikawa and Chou have developed methods such as the mosaic model and the fiber undulation model [4]. Closed form methods developed at NASA Lewis for laminated composites include methods such as the simplified micromechanics developed by Chamis and associates [5-7] and the generalized method of cells developed by Aboudi and Pindera [8].

In order to verify the closed-form analytical methods, and to examine local behaviors such as microstresses, advanced finite element methods have been utilized to analyze micromechanical behavior [1]. With these methodologies, appropriate representative volume elements are explicitly modeled and discretized with appropriate meshes and boundary conditions, and displacements. stresses and strains are directly computed. One classic example of this methodology for laminated composites is Dvorak's periodic hexagonal array [9]. Composite micromechanical analyses utilizing the finite element method have been carried out at NASA Lewis (examples include Refs [10-12]). As discussed in Ref. [13], Whitcomb has applied finite element techniques to the analysis of plain weave woven composites.

With the success of the application of finite element methods to composite micromechanical modeling as a motivation, a joint program between the State University of New York at Buffalo and NASA Lewis was established to examine the possible application of the boundary element method to composite micromechanical analysis. The motivation behind using the boundary element method was the ability of BEM to model a three-dimensional structure with surface discretization only, which could be an advantage in developing complex composite unit cell models.

This paper discusses some of the details of the new boundary element techniques, and presents some example applications where the boundary element method has been utilized to compute effective properties of actual materials. Several examples involving laminated composites are presented in order to verify these boundary element techniques by solving relatively simple problems which are easy to generate. and for which alternative solutions are available. By comparing the boundary element results to experimental observations and results obtained by using established alternative analytical and finite element methods, the accuracy of the boundary element 
methods used here can be established. A simple example involving a $2-\mathrm{D}$ plain weave woven composite is also discussed. While several other methods exist to analyze this particular woven architecture, we hope that the model developed and discussed here can be expanded to simulate more complex architectures, such as 3-D weaves and braids, for which alternate analytical methods may not be available. The objective of this paper is to demonstrate that the boundary element method has the potential to be effectively used in conducting composite micromechanical analyses.

\section{ANALYSIS METHODOLOGY}

The computerized tool BEST-CMS (boundary element solution technology-composite modeling system) [14-16] is utilized to conduct the boundary element analyses presented in this paper. BEST - CMS includes the capability to conduct elastostatic, heat conduction and thermoelastic analyses. Full details of the derivation of the method are given in the above cited references, but a few relevant details of the formulation for steady state heat conduction and elastostatic analyses are given below.

The formulation begins with a boundary integral equation describing the displacement (or temperature) at a point $\xi$ inside a homogeneous material with holes

$$
\begin{aligned}
C_{i j}(\xi) u_{i}(\xi)= & \int_{s}\left[G_{i,}^{\mathrm{o}}(x, \xi) t_{i}^{\prime \prime}(x)\right. \\
& \left.-F_{i j}^{\mathrm{o}}(x, \xi) u_{i}^{\prime \prime}(x)\right] \mathrm{d} S(x) \\
& +\sum_{n=1}^{N} \int_{S^{\prime}}\left[G_{i j}^{\mathrm{H}}(x, \xi) t_{i}^{\prime \prime}(x)\right. \\
& \left.-F_{i l}^{\mathrm{H}}(x, \xi) u_{i}^{H}(x)\right] \mathrm{d} S^{N}(x)
\end{aligned}
$$

where $G_{i j}$ and $F_{i}$ are the fundamental solutions of the governing differential equations of the matrix of infinite extent, $C_{i j}$ are constants determined by the geometry at $\xi, u_{i}, t_{i}$ are displacements and tractions (or temperature and flux). $S . S^{n}$ are the surfaces of the outer boundary of the matrix and the $n$th hole, respectively, $N$ is the number of individual holes in the matrix. Superscripts $O$ and $H$ identify quantities on the outer surface of the matrix and the outer surface of the hole, respectively.

To simulate a composite material, one desires to fill each of the holes with solid material (i.e. put fibers into the matrix holes). The boundary integral equation describing the displacement (or temperature) at a point $\xi$ in each of the $N$ fibers can be written as follows:

$$
\begin{aligned}
C_{i j}^{\mathrm{l}}(\xi) u_{i}(\xi)= & \int_{s^{n}}\left[G_{i j}^{\mathrm{l}}(x, \xi) t_{i}^{1}(x)\right. \\
& \left.-F_{i j}^{\mathrm{l}}(x, \xi) u_{i}^{1}(x)\right] \mathrm{d} S^{n}(x)
\end{aligned}
$$

where $G_{i j}^{1}$ and $F_{i j}^{1}$ are the fundamental solutions of the $n$th fiber, $C_{i j}^{1}$ are constants determined by the geometry at $\xi$ in insert $n, u_{i}^{1}, t_{i}^{1}$ are displace. ments and tractions (or temperature and flux) associated with the $n$th insert, $S^{N}$ is the surface of the $n$th insert.

For the condition where the insert is perfectly bonded to the fiber, the displacement (or temperature) of the matrix and fiber at the fiber-matrix interface are set equal and the tractions (or fluxes) of the matrix and fiber are set to be equal and opposite. Furthermore, Banerjee et al. [14-16] postulated that the Poisson's ratio of the fiber can be set equal to the Poisson's ratio of the matrix as a result of the elastic modulus of the fiber usually being much greater than that of the matrix. Test cases showing the applicability of this assumption are shown in Ref. [16]. This assumption leads to the following relation involving the $F_{i j}$ kernels

$$
F_{i j}^{\mathrm{l}}(x, \xi)=-F_{i \prime}^{\mathrm{H}}(x, \xi)
$$

By applying these assumptions to eqn (2), the following modified boundary integral equation for the $n$th insert is obtained

$$
\begin{aligned}
C_{i j}^{\mathrm{l}}(\xi) u,(\xi)= & \int_{u^{n}}\left[-G_{i j}^{\mathrm{I}}(x, \xi) t_{i}^{\mathrm{H}}(x)\right. \\
& \left.+F_{i j}^{\mathrm{H}}(x, \xi) u_{i}^{\mathrm{H}}(x)\right] \mathrm{d} S^{n}(x) .
\end{aligned}
$$

By adding the $N$ fiber eqn (4) to eqn (1), the modified boundary integral equation for a matrix material with fibers is obtained

$$
\begin{aligned}
& \bar{C}_{\eta \prime}(\xi) u_{i}(\xi)=\int_{s}\left[G_{i i}^{\mathrm{o}}(x, \xi) t_{i}^{0}(x)\right. \\
& \left.-F_{u j}^{o}(x, \xi) u_{,}^{\circ}(x)\right] \mathrm{d} S(x) \\
& +\sum_{n=1}^{N} \int_{S^{*}}\left[\overline{G_{n}^{n}}(x, \xi) t_{i}^{H}(x)\right] \mathrm{d} S^{N}(x)
\end{aligned}
$$

In discretizing eqns (4) and (5), a unique formulation is employed in modeling the fibers in order to avoid the necessity of a fine discretization of the fiber. Specially formulated "Fiber Elements" are utilized in which only the centerline of the fiber is defincd, using nodes and elements, and the fiber radius is defined at each node. Both straight and curvilinear fibers are permitted, however, all fibers are assumed to have a circular cross-section along the entire length. The fiber surfaces and the variation of the field variables in the plane of the fiber cross-section are represented through the use of trigonometric circular shape functions and closed form analytical expressions within the boundary element formulation. To calculate the variation of the field variables along the length of the fiber, numerical integration is performed. 
Specifically, the circular shape functions are employed to approximate the variation in the traction (or flux) around the circumference of the fiber in order to convert the two-dimensional surface integral of the insert to a one-dimensional integration. The modified traction equations are then incorporated into the integral within the summation in eqn (5), utilizing a local coordinate system with its center at the center of the fiber and the $z$-axis aligned with the fiber centerline. The last term in eqn ( 5 ) thus becomes

$$
\begin{aligned}
& \int_{S m}\left[\overline{G_{i j}^{n}}(x, \xi) t_{i}^{\mathrm{H}}(x)\right] \mathrm{d} s^{m}(x) \\
& =\int_{C m} a_{j k} \int_{0}^{2 \pi} \overline{G_{l k}^{\text {local }}}(R, \theta, z, \bar{\xi}) M^{i} R \mathrm{~d} \theta a_{i l} \ell_{i} \mathrm{~d} C^{m} \\
& =\int_{C m} G_{i j}(R, z, \bar{\xi}) t_{i} \mathrm{~d}^{m}(z)
\end{aligned}
$$

where the integration over $C^{m}$ is now a onedimensional curvilinear integration along the fiber, $G_{i j}$ is the analytically integrated fiber kernels, $\gamma$ varies from $1,2,3, a_{j k}$ is the transformation matrix and $\boldsymbol{M}^{i}$ is the shape function. Similar analytical integration is caried out in eqn (4).

BEST-CMS includes provisions to simulate mechanical and thermal fiber-matrix interface behavior. The interface behavior (including perfect bonding, sliding interfaces, linear spring interfaces, thermal resistance interfaces and progressive debonding with gap openings and frictional slipping) is incorporated directly into the boundary integral formulations by adjusting the displacement continuity relation so that instead of the matrix and fiber displacements being equal at the interface, the following relationship is utilized [16]:

$$
u_{i}^{\mathrm{H}}(x)=u_{i}^{1}(x)+d_{i}(x)
$$

where $d_{i}$ is the difference between the displacement (temperature) of the fiber and the displacement (temperature) of the matrix. By utilizing this relation. eqns (4) and (5) are appropriately modified [16]. For linear spring interfaces, spring constants normal and parallel to the fiber are defined. The thermal interface is modeled by defining a thermal resistance value which relates the heat flux across the interface to the temperature difference between the fiber and matrix.

As mentioned in the introduction, the motivation behind this work is the success that has been achieved in using the finite element method to conduct composite micromechanical analyses. Finite element analyses have proven useful in primarily two areas: verification of equivalent properties computed by closed form analytical methods, and determination of the magnitude and location of local stress and strain concentrations. However, while for unidirectional (or bidirectional) laminates a finite element mesh is not overly difficult to construct, for more complex architectures such as angle-plied laminates or woven composites (particularly 3-D weaves and braids), the construction of an appropriate finite element mesh becomes much more complex and time consuming. The boundary element methods used in BEST-CMS provide a method to reduce the mesh complexity of a three-dimensional composite micromechanical model, particularly for complex architectures. The composite matrix is modeled by discretizing only the outer surface, which eliminates the need for an interior volumetric mesh. Additionally, by modeling the fibers using line elements (due to the one-dimensional numerical integration discussed above), the modeling of a complex fiber architecture can be simplified. To incorporate a fiber-matrix interface into the composite model, the interface parameters are directly entered into the BEST-CMS input file, eliminating the need to explicitly generate gap or three-dimensional interface elements. Again, while for simple composite architectures these modeling simplifications may be trivial if at all applicable, for complex architectures the time and effort saved in the generation of a composite micromechanical model has the potential to be significant.

Several other potential advantages of the boundary element techniques used in BEST-CMS, while still being refined and/or tested and therefore not presented in the results to follow, are worth mentioning. First, the concurrent thermoelastic formulations allow a thermal analysis and a stress analysis to be conducted simultaneously, thus eliminating the need to first conduct a thermal analysis to obtain temperature distributions, and then conducting a stress analysis. Also, eqns (4) and (5) can potentially be solved at any point in the interior of the matrix or fiber, allowing the precise calculation of interior displacements and stresses.

There are several limitations to the current formulation and implementation of the boundary element methods in BEST-CMS and the BEST-CMS code itself which should be discussed. Fiber ends as free surfaces cannot be represented due to the insert element formulation, which results in the fibers lying entirely within the matrix outer surface. The primary effect of this assumption is that loads and heat flow must be transferred from the outer surface of the matrix to the fiber. The effects of this limitation and potential methods of overcoming the limitation vary with the type of analysis undertaken. For example, for elastic analyses with a weak fiber-matrix interface, the spring constant parallel to the fiber must be set to a large value to prevent the matrix being pulled away from the fiber under load. Additionally, for thermal analyses, when the thermal conductivity of the matrix is much lower than that of the fiber, when a temperature gradient parallel to the fiber is applied, the heat flux values on the outer boundary may be lower than expected. Another assumption within the insert element formulation is that the cross-section of 
the fiber elements is currently restricted to a circular shape. For angleplied laminates, this assumption requires care to be taken in properly placing-spacing the fibers within the matrix in order not to seriously reduce the effective fiber volume fraction. Additionally, for woven composites, where the fiber tows have a definite elliptical shape, multiple fiber elements must be used to represent a fiber tow in order to correctly approximate the shape.

There are currently several limitations to the BEST-CMS code itself separate from the assumptions in the formulations which currently affect how results are computed. All of these effects have been noted and hopefully will be eliminated in future versions of the code. First, rigid plane boundary conditions are not specifically available within BEST-CMS. Specifically, since nodal tying and other multi-point constraints cannot be applied to the boundary element model, when computing effective properties nodal averages of quantities such as displacement and temperature must be taken. Any results obtained on the boundary should be compared to interior results in order to determine the accuracy of the boundary results. Another restriction that affects these analyses is that currently the fiber-matrix interface formulations are not available for a thermoelastic analysis with multiple time steps. As will be discussed below, this restriction limits the ability of the code to simulate the full behavior of a material under residual stresses that debonds once the residual stresses are overcome. Additionally, computational speed is currently not competitive with commercial finite element codes. The lack of computational speed is due both to the fully populated matrices inherent to the boundary element method, as well as the fact that, since BEST-CMS is still primarily a research code, the computational efficiency issues have not been addressed to any great extent. Hopefully, in future versions of BEST-CMS the computational efficiency can be improved.

Several example applications of the boundary element methods presented here are discussed below. While the examples presented are fairly simplistic and can be analyzed by a variety of methods (perhaps more quickly and efficiently), they provide a basis for demonstrating BEST-CMS. Examples are presented for laminated composites where a number of experimental and/or alternate analytical results are available with which to check the accuracy of the boundary element results (using relatively simple boundary element models). The example utilizing the 2-D weave presents an area where we feel utilizing the boundary element technique may be most beneficial, that of woven and braided architectures. Although the 2-D weave can be analyzed using a variety of methods, we hope that the techniques and boundary element models developed to analyze a 2-D weave can be expanded to more complex architectures.
Table 1. Constitutive properties for elastic analyses of laminated composites

\begin{tabular}{lcc}
\hline Material & Modulus (GPa) & Poisson's ratio \\
\hline SiC & 390 & 0.19 \\
Ti-15-3 & 88 & 0.32 \\
RBSN & 110 & 0.22 \\
\hline
\end{tabular}

\section{ELASTIC ANALYSIS OF LAMINATED COMPOSITES}

The first set of analyses presented examines the ability of BEST-CMS to simulate the elastic behavior of a laminated composite material. The first composite system examined is composed of $\mathrm{SiC}$ (SCS-6) fibers with a fiber diameter of $145 \mu \mathrm{m}$ embedded within a titanium alloy (Ti-15V-3Cr-3Sn-3Al or Ti-15-3 for short) matrix, with a fiber volume fraction of 0.34 . The material properties of the fiber and matrix, obtained from Ref. [17], are listed in Table 1.

As discussed in Refs [17] and [11], for this material residual stresses are imposed during processing. The computational results discussed in the references seem to indicate that one major effect of these residual stresses is in controlling the behavior of the fiber-matrix interface. Specifically, until a tensile load is applied which is of sufficient magnitude to overcome the residual stresses, the interface behaves as perfectly bonded and the composite behaves as a linear elastic material. Once the interface debonds, the stress states imposed by the residual stresses appear to affect the nonlinear (inelastic) regimes of the material behavior.

The boundary element results discussed here only deal with the effective modulus for the initial range of the material stress-strain behavior, in which the residual stresses cause the interface to behave as a perfect bond and the overall material behavior can be modeled as linear elastic. Residual stresses are not explicitly applied, but their effects are implicitly modeled in the specification of a perfect fiberinterface bond. Future studies will involve simulating the full range of material behavior, including explicitly applying residual stresses and then progressively applying a tensile load until the interface debonds and the matrix material eventually yields. Unfortunately, the current version of BEST-CMS does not allow complex fiber-matrix interface conditions to be combined with a thermoelastic analysis with multiple time steps, which precludes such an analysis from being conducted at this time.

The boundary element model utilized for these analyses is a four cell square model, where the model thickness equals the width. Figure 1 shows a sample boundary element model for a unidirectional [0] composite, with the fiber and matrix elements labeled. Eight noded quadrilateral elements are used to model the composite matrix, and three noded line elements are used to model the fibers. Roller nodal constraints are applied to the back $(y-z)$, left $(x-z)$ and bottom $(x-y)$ faces of the model, and a uniform pressure load 


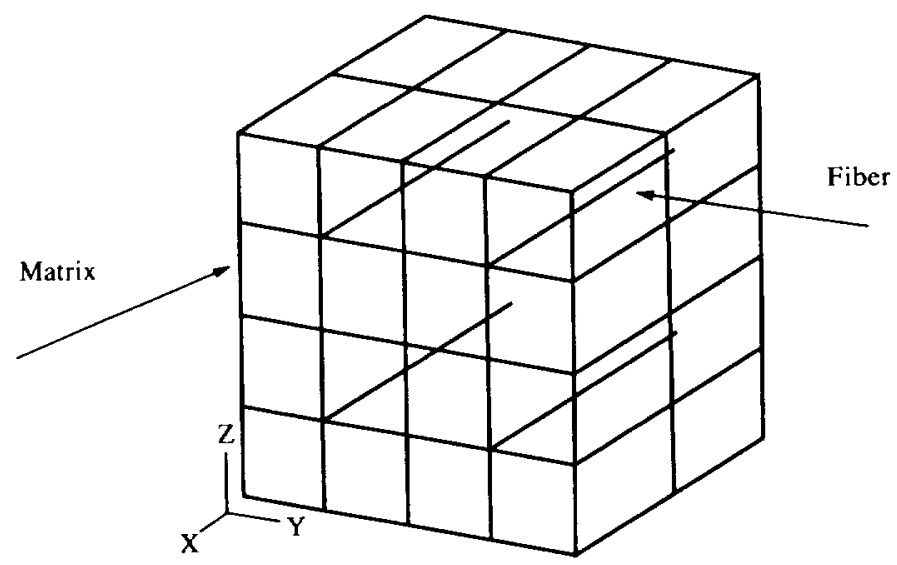

Fig. 1. Sample boundary element model for [0] laminated composite.

is applied to the front $(y-z)$ face. The effective composite modulus is then calculated by computing the average displacement across the model face where the pressure load is applied for a given stress level, and dividing by the model thickness to obtain the equivalent strain. While doing these calculations across an interior cross-section would have been preferable, due to the fact that the BEST-CMS routines to compute values in the interior of the material are still being refined, the boundary displacement values needed to be used.

The results obtained for the initial elastic tensile modulus are displayed in Fig. 2 for [0] and [90] laminate orientations. The boundary element results are compared to experimental values obtained by Lerch and Saltsman [17] and three-dimensional finite element results obtained using the NASTRAN [18] program. The boundary element results are within $10 \%$ of the experimental observations for both laminate orientations, and the boundary element results are reasonably close to the finite element values. The larger discrepancy seen for the [0] laminate between the boundary element and finite element values is most likely due to the boundary element assumption that fiber ends are not free surfaces. Since the matrix is softer than the fiber, and the loads must be transferred from the matrix to the fiber in the boundary element analysis, it is reasonable to expect that on the boundary of the matrix the displacements would be larger for a given stress level (thus reducing the effective modulus) than for the finite element model, where the fiber extends all the way to the outer surface. Refining the boundary element mesh may also serve to help improve the results, since a relatively coarse boundary element mesh was utilized. For the [90] laminate, where the loads are applied perpendicular to the fiber, the fiber end assumption plays a much less significant role in the boundary element results, and thus the boundary element and finite element results are much closer to each other. Still, a reasonably good match is obtained between the boundary element results and the experimental and finite element values, for a relatively coarse boundary element mesh.

The next laminated composite system to be examined consists of $\mathrm{SiC}$ (SCS-6) fibers embedded within a reaction bonded silicon nitride ( $\mathrm{RBSN}$ ) matrix with a fiber volume ratio of 0.30 . The material properties

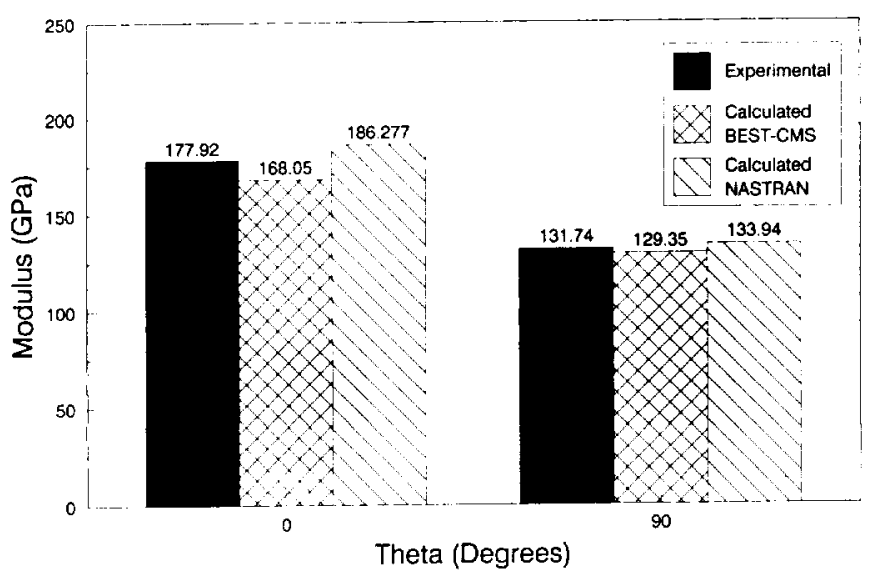

Fig. 2. Effect of fiber orientation angle on effective initial longitudinal tensile modulus for $\mathrm{SiC}$ Ti-15-3. 


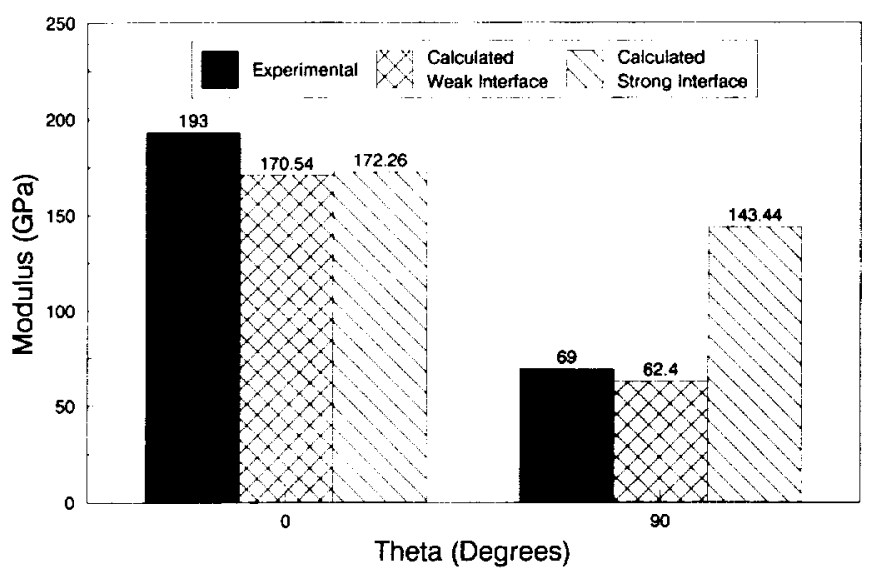

Fig. 3. Effect of fiber orientation angle and fiber-matrix interface on effective initial longitudinal tensile modulus for SiC-RBSN.

are as given in Table 1 [19]. For this material, the fiber-matrix interface is weakly bonded in the full range of the material stress-strain behavior, including the initial region, due to the imposed residual stresses. The material can be considered to be linear elastic in the initial portions of the material behavior [19].

The effective modulus and Poisson's ratio for the initial range of the material behavior are computed for this material. To model the fiber-matrix interface for this material, a linear spring interface is used. As noted in Ref. [7], for a composite with a weak interface, the behavior parallel to the fiber does not depend on the interfacial conditions. If the fiber surfaces of the boundary element model extended to the outer surface of the model and multi-point constraint conditions (nodal tying) were available, this condition would be trivially satisfied. However, since the fiber surfaces do not extend to the outer surface in BEST-CMS, the spring constant parallel to the fiber must be set to a very large (near infinity) value, in order to prevent the matrix from pulling away from the fiber in the direction parallel to the fiber.
Since the interface is weakly bonded, the spring constant in the direction normal to the fiber is set to a negligible (near zero) value. The boundary element model and boundary conditions for these analyses are the same as was used previously. The results are again computed for [0] and [90] laminates.

The computed modulus results are shown in Fig. 3 and the computed Poisson's ratio results are shown in Fig. 4. The boundary element results obtained by using the imposed interface conditions are compared to experimental values obtained by Bhatt and Phillips [19]. To confirm-verify that the boundary element analyses are correctly capturing the fiber-matrix interface behavior, boundary element results computed by using a perfect (strong) fiber-matrix bond are also plotted.

Examining the results, the computed boundary element results computed using a weak interface are within approximately $10 \%$ of the experimental values. Several points of interest can be noted in the results. First, for the [0] laminate the fiber-matrix interface has a very small effect on the calculated

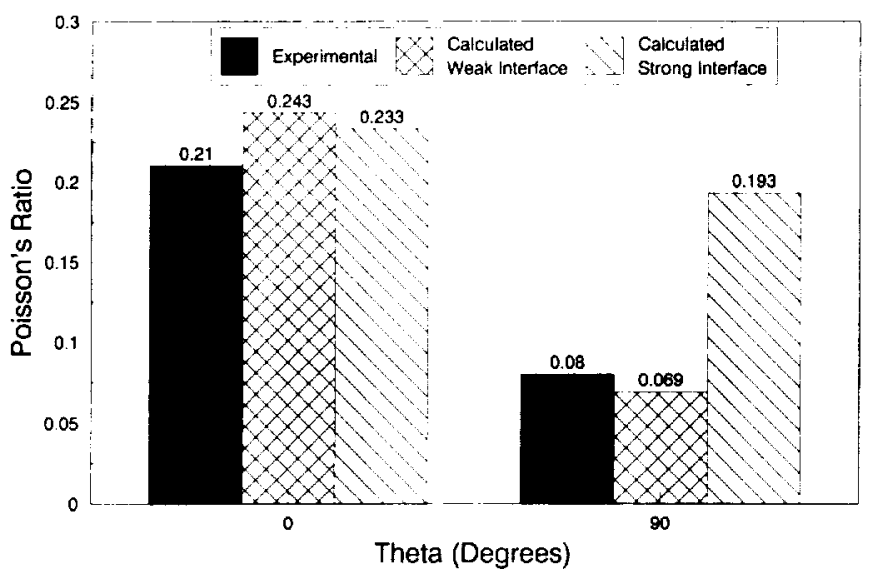

Fig. 4. Effect of fiber orientation angle and fiber-matrix interface on effective initial Poisson's ratio for SiC-RBSN. 
parameters. This result is to be expected since the condition was imposed that the interface was not supposed to affect the composite behavior in the direction parallel to the fiber. However, for the [90] laminate, the boundary element results computed using a strong fiber-matrix interface significantly overpredict the composite properties, and are significantly different from the results computed using a weak interface. The imposed interface, which allows the fiber to separate from the matrix in the direction normal to the fiber, is thus necessary in order to correctly model the effective properties of this material. Another check on the results is that the boundary element results computed for both strong and weak interfaces closely match the results reported in Ref. [7], which were computed using Chamis' simplified micromechanics.

As with the $\mathrm{SiC}-\mathrm{Ti}-15-3$, the computed results for the [90] laminate are closer to the experimental values than the results obtained for the [0] laminate. Again, this discrepancy is most likely to be due to the fiber end assumption inherent within the boundary element formulation, which causes the loads to be transferred through the soft matrix to the fiber for a loading parallel to the fibers. Mesh refinement may also help to improve the boundary element results, as again a relatively coarse boundary element mesh was used.

\section{THERMAL ANALYSIS OF LAMINATED COMPOSITES}

The next set of analyses examines the ability of BEST-CMS to simulate the steady state thermal (heat conduction) behavior of a laminated composite. For these analyses, the effective longitudinal room temperature thermal conductivity for [0] and [90] laminates is the material property of interest. Bhatt et al. [20] examined a system consisting of $\mathrm{SiC}$ (SCS-6) fibers embedded in a reaction bonded silicon nitride (RBSN) matrix, but, due to differences in processing, there are some differences between this specific material setup and the material setup for SiC-RBSN that was described in the previous section. Two different kinds of samples were utilized in the experimental studies discussed in Ref. [20]. First, one set of samples, with a fiber volume fraction of 0.32 , was made in which a carbon-rich coating was placed around the fibers. The carbon-rich coating caused a weak fiber-matrix interface to exist after processing was completed. Fiber and matrix constitutive thermal properties are given in Table 2 [20]. Another set of samples, with a fiber volume fraction

Table 2. Constitutive thermal properties for thermal analyses of laminated composites

\begin{tabular}{lc}
\hline Material & Conductivity $(\mathrm{W} / \mathrm{mK})$ \\
\hline SiC & 22.5 \\
RBSN (uncoated fibers) & 11.75 \\
RBSN (coated fibers) & 4.2 \\
\hline
\end{tabular}

of 0.38 , was manufactured that had no fiber coating. The lack of fiber coating caused the fiber-matrix interface bond to remain strong even after processing. Another difference for the material with uncoated fibers is that, due to differences in processing, the matrix density was significantly higher than for the case with uncoated fibers, which caused the matrix conductivity for the material with uncoated fibers to be more than twice that of the material with coated fibers. Fiber and matrix constitutive properties for the material with uncoated fibers are also given in Table 2 [20].

The differences in interface behavior based on fiber coating were found to have a significant effect on the effective composite thermal conductivity values determined experimentally in Ref. [20]. Specifically, for the material with uncoated fibers, the material remained strongly bonded during heating and there was no thermal resistance found between the fiber and the matrix. For the material with coated fibers, however, the interface was weak, and the longitudinal thermal conductivity value for a [90] laminate was much lower than the value which was computed using a closed-form micromechanical formulation assuming no thermal interface. The authors of [20] deduced that the weak interface caused an interfacial gap to form between the fiber and matrix, which effectively placed a thermally resistant barrier between the fiber and matrix when heat flow normal to the fibers was applied. Alternatively, when heat flow was applied parallel to the fiber direction, the interfacial gap had no effect on the thermal conductivity values, which is reasonable based on the observations made in Ref. [7] that interfacial conditions have an insignificant effect on composite properties in the direction parallel to the fiber.

The boundary element model used for these analyses is that shown in Fig. 1. The parameters used to model the fiber-matrix interface were varied based on which material was being simulated (uncoated or coated fibers). For the material with uncoated fibers, since the material was found experimentally to remain strongly bonded, no thermal interface was applied, and the heat flow between fiber and matrix was assumed not to be impeded in any way. For the material with coated fibers, however, a thermal interface was applied between the fiber and matrix in order to simulate the effects of the weak bonding and interfacial gap which were noted in the experiments. In the direction parallel to the fiber, the thermal resistance value was set to a negligible (effectively zero) value. As noted above in the interface discussions for the mechanical loading, theoretically the interface behavior should not affect the effective property computations in the direction parallel to the fiber [7], but the fiber end assumption (fiber ends are not free surfaces) in BEST-CMS results in the heat flow being transferred from the matrix to the fiber. If a thermal resistance was applied in the direction parallel to the fiber, a reduced heat flow would be 


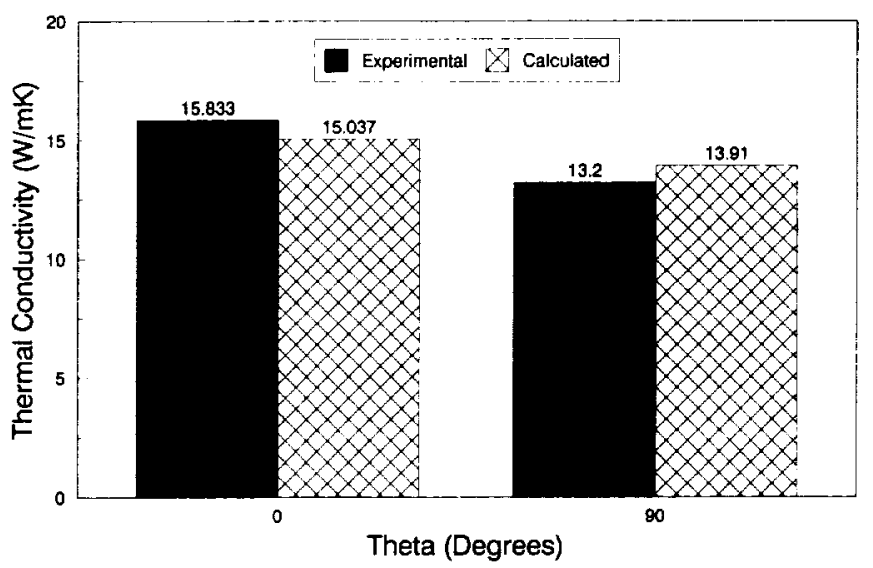

Fig. 5. Effect of fiber orientation angle on room temperature effective thermal conductivity for SiC-RBSN with uncoated fibers.

transferred to the fiber. In the direction normal to the fibers, a very strong thermal resistance (near infinity) was applied to simulate the thermal barrier caused by the interfacial gap. The applied interfacial conditions matched the experimental behavior observed when the specimen with coated fibers was tested in vacuum, in which the thermal resistance was found to be the strongest (almost total blocking of heat flow between the fiber and matrix). To compute the effective thermal conductivity, a $100^{\circ} \mathrm{C}$ temperature gradient was applied, and the equivalent (average) resulting heat flux was computed.

The equivalent thermal conductivity results computed using the boundary element method, along with the experimental values [20] for comparison are plotted in Fig. 5 for the material with uncoated fibers and in Fig. 6 for the material with coated fibers. For the material with coated fibers, results computed using no thermal interface are also plotted to examine-confirm the effects of applying the thermal interface. Examining the results, for the material with uncoated fibers the boundary element results are reasonably close to the experimental values, indicating that the model is reasonably close to simulating the actual material behavior. For the material with coated fibers, several items in the results are worth noting. First, for the thermal conductivity computed by using a thermal interface, while the result for the [90] laminate compares favorably to the experimental value, for the [0] laminate the computed result underpredicts the experimental value. This discrepancy is the result of the BEST-CMS fiber end assumption. Since the fiber ends are not free surfaces, the heat flow must be transferred from/to the matrix to/from the fiber. For this particular case, since the thermal conductivity of the matrix is significantly lower than that of the fiber, the heat flow transferred to and from the fiber is reduced from what would be the case if the fiber extended to the model outer surface. For the [90] laminate, the comparison of the computed value to the experimental value is much more favorable, since the fiber end assumption is much less critical

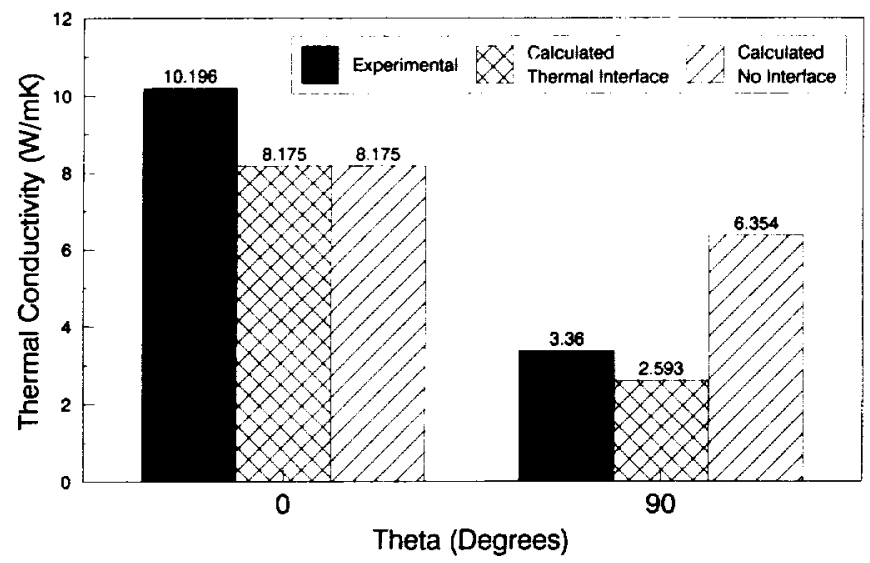

Fig. 6. Effect of fiber orientation angle and thermal interface on room temperature effective thermal conductivity of $\mathrm{SiC}-\mathrm{RBSN}$ with coated fibers. 

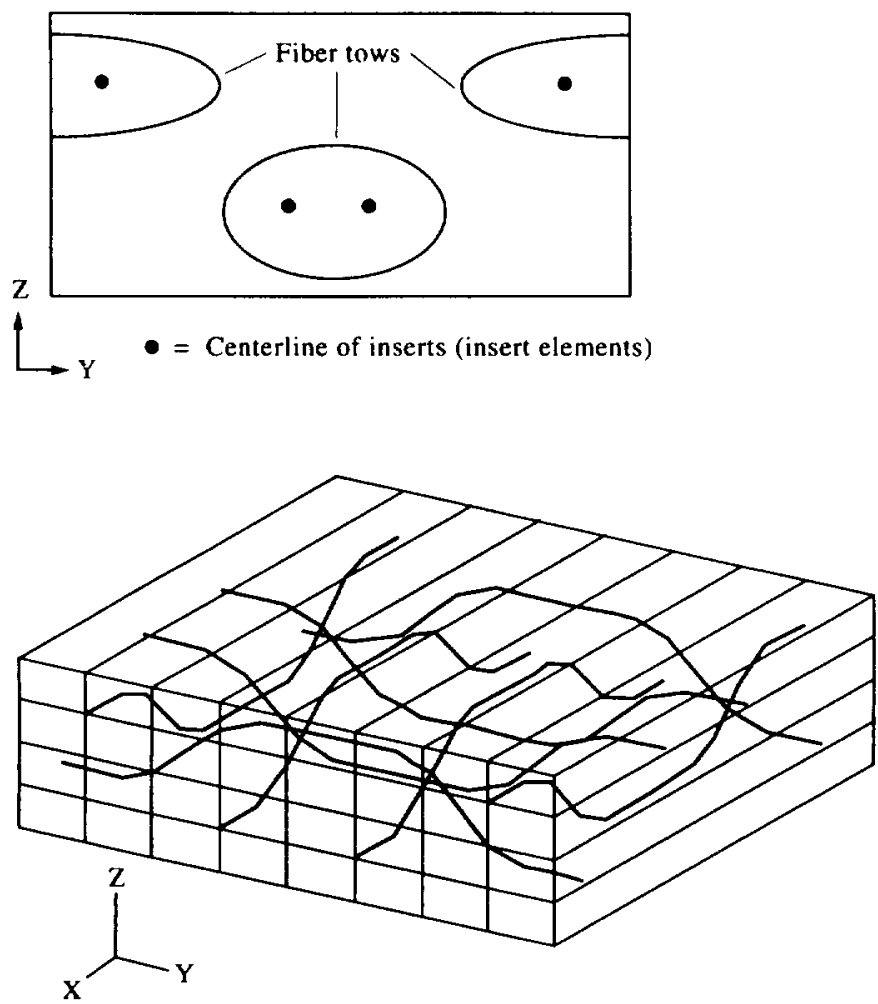

Fig. 7. Sample boundary element model for plain weave $[0 / 90]$ woven composite.

when the heat flow is applied normal to the fiber direction. Another point of interest is that for the [90] laminate the thermal conductivity computed using a thermal interface is significantly lower than the value computed using no interface, and compares much more favorably to the experimental value. The values computed for the [0] laminate for both interface conditions match, but that was a condition which was imposed in order to attempt to match the actual material behavior. The values computed for the [90] laminate, however, indicate that including an appropriate thermal interface is necessary in cases where a thermal barrier between the fiber and matrix exists in the actual material, and that the thermal interface capability in BEST CMS does a reasonable job in simulating this behavior.

\section{ELASTIC ANALYSIS OF WOVEN COMPOSITES}

The final example problem presented in this paper involves examining the ability of BEST-CMS to compute the initial (linear) effective longitudinal modulus of a 2-D plain weave woven composite. It is in the area of woven composites that the boundary element formulations presented here have the greatest potential to provide a useful analytical technique. To construct a full finite element micromechanical model of even a 2-D plain weave woven composite would be very complex and time consuming. When modeling a 3-D woven or braided structure, as we hope to do, constructing an appropriate finite element model would increase the model complexity to an even greater degree.

The boundary element model that was used to model the $2-\mathrm{D}$ plain weave $[0 / 90]$ woven composite analyzed in this study is shown in Fig. 7. There are several points of interest to note in this model. First, eight-noded quadrilateral elements were once again utilized to model the matrix outer surface. To model the fiber lows, curvilinear fiber elements were used. In an actual woven composite, the fiber tows have a definite elliptical shape. However, in BEST-CMS the fiber elements are assumed to have a circular crosssection. In order to account for this assumption and still make some effort to correctly model the composite microstructure, two fiber elements were utilized to model each fiber tow. While using two fiber elements for each fiber tow adds to the model complexity, it is hoped that by attempting to model the correct fiber tow geometry more accurate results can be obtained.

In actual woven ceramic matrix composites, the material porosity both within the matrix and between the individual fibers in each fiber tow significantly affects the overall material behavior and must be accounted for in an analytical model. For this study, since only overall effective properties for the initial linear range of the material behavior were computed, a very simple first approximation was used to account for the porosity in the boundary element model. Specifically, the fiber elements (and their associated 


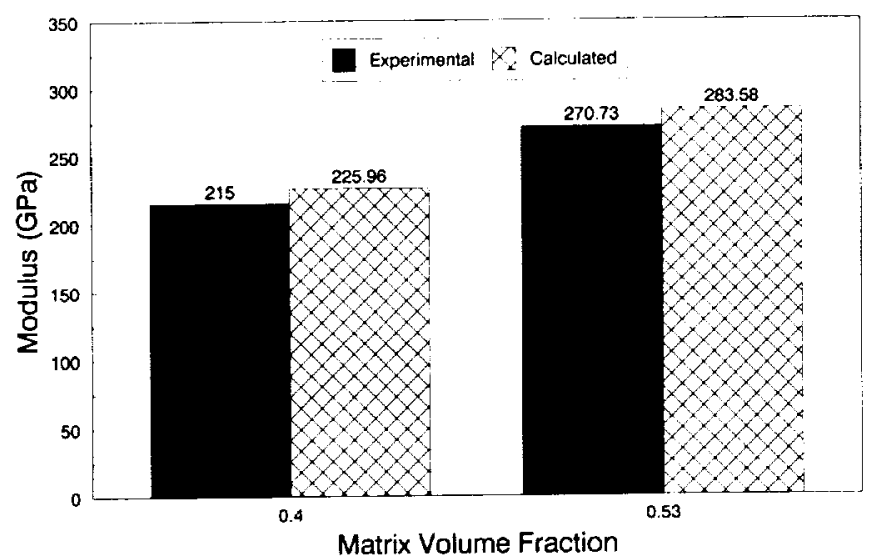

Fig. 8. Effect of matrix volume fraction on initial longitudinal tensile modulus for 2-D [0/90] plain weave Nicalon-SiC.

properties) are explicitly defined in the boundary element model using the given fiber volume fraction. The remainder of the model is then assumed to consist of matrix and pores. To compute the effective properties of the "matrix" (consisting of matrix and pores) a very simple rule of mixtures approximation was used in which the matrix properties and matrix volume fraction and a pore modulus of zero were used. Again, since the actual pores are distributed throughout the model material, to accurately examine local effects or overall material behavior in anything other than the linear elastic portion of the stress-strain curve, a more sophisticated technique for incorporating the material porosity into the boundary element models will have to be devised.

The specific material considered for this study consists of Nicalon fiber tows embedded in a silicon carbide matrix, with constituent material properties as given in Table 3 . The fiber volume fraction is set to 0.40 , and two matrix volume fractions are considered, 0.40 ( $20 \%$ porosity) and 0.53 ( $7 \%$ porosity), in order to examine the ability of BEST-CMS to simulate the effects on the longitudinal tensile modulus of varying the material porosity (at least to a first approximation level). The boundary conditions applied to the model are similar to those used for the elastic analyses of laminated composites.

The longitudinal modulus results for each of the two materials (with the two different matrix volume fractions) are plotted in Fig. 8. The boundary element results are compared to experimental values obtained from Refs [21] and [22]. As can be seen from the figure, for both material conditions the boundary element results are within approximately $5 \%$ of

Table 3. Constitutive properties for elastic analysis of woven composites

\begin{tabular}{lcc}
\hline Material & Modulus (GPa) & Poisson's ratio \\
\hline Nicalon & 200 & 0.25 \\
SiC matrix & 350 & 0.2 \\
\hline
\end{tabular}

the experimental values. In addition, the boundary element results reflected the experimental trend that as the material porosity was decreased, the longitudinal modulus increased (which is an expected result). Considering all of the approximations that were used in this analysis, this comparison appears to be fairly good. The results obtained here seem to indicate that the boundary element formulations presented here have the ability to model woven composite architectures. Attempts will now be made to expand the woven composite models to more complicated architectures, such as 3-D weaves and braids, and to model more complex local and nonlinear behaviors.

\section{CONCLUSIONS}

A new boundary element formulation for the micromechanical analysis of composite materials has been presented and its applicability examined through the discussion of several example problems. For the applications presented, the boundary element results for the most part compared reasonably well to experimental values and/or results obtained through alternate analytical methods. For the cases where the comparison was not quite as favorable, possible reasons for the discrepancies relating both to the theoretical formulation and specific deficiencies of the BEST-CMS computer code were identified. However, while some issues still need to be addressed to make the code more robust the BEST-CMS code may provide a viable alternative, particularly for complex woven composite architectures, to traditional finite element techniques for composite micromechanical analyses.

\section{REFERENCES}

1. A. K. Noor and R. S. Shah, Effective thermoelastic and thermal properties of unidirectional fiber-reinforced composites and their sensitivity coefficients. Compos. Struct. 26, 7--23 (1993).

2. C. J. Lissenden and C. T. Herakovich, Comparison of 
micromechanical models for elastic properties. Engineering, Construction and Operations in Space III, Space 92 Proc. Third Intermational Conference ASCE, $1309-1322$ (1992)

3. J. Aboudi. Mechanics of Composite Materials, A Unified Micromechanical Approach. Elsevier Science, New York (1991).

4. T.-W Chou and F. K. Ko, Textile Structural Composites. Elsevier Science, New York (1989).

5. P. L. N. Murthy and C. C. Chamis, Integrated composite analyzer (ICAN) users and programmers manual. NASA TP-2515 (1986).

6. D. A. Hopkins and C. C. Chamis. A unique set of micromechanical equations for high temperature metal matrix composites. NASA TM-87154 (1985).

7. S. K. Mital. P. L. N. Murthy and C. C. Chamis, Ceramic matrix composites properties microstresses with complete and partial interphase bond. NASA TM-106136 (1993).

8. J. Aboudi and M.-J. Pindera. Micromechanics of metal matrix composites using the generalized method of cells model: user`s guide. NASA CR-190756 (1992).

9. J. L. Teply and G. J. Dvorak, Bounds on overall instantaneous properties of elastic plastic composites J. Mech. Phys. Solids 36, 2958 (1988).

10. S. K Mital, J. J. Caruso and C. C. Chamis. Metal matrix composites microfracture: computational simulation. NASA TM-103153 (1990).

11. B. A. Lerch, M. E. Melis and M. Tong. Experimental and analytical analysis of stress strain behavior in : [90 0$] . \mathrm{SiC} \mathrm{Ti}-15-3$ laminate. NASA TM- 104470 (1991).

12. J. G. Sanfeliz. Elastic plastic finite element analyses of an unidirectional, $9 \mathrm{vol} \%$ tungsten fiber reinforced copper matrix composite. NASA TM-106304 (1993)
13. J D Whitcomb, Three-dimensional stress analysis of plain weave composites. NASA TM-101672 (1989).

14. P. K. Banerjee, D. P. Henry, Jr and G. F. Dargush, Micromechanical studies of composites by BEM. Proc. ASME Conf. Enhancing Analysis Techniques For Composite Materials, NDE-Vol. 10, 221-228 (1991).

15. P. K. Banerjee and D. P. Henry. Micro and macromechanical analyses of composites by BEM. Proc. ASME Conf. Constitutive Behatior of High-Temperature Composites. MD-Vol. 40, 116 (1992).

16. D. P. Henry, P. K. Banerjee, G. F. Dargush. D. A Hopkins and R. K. Goldberg, BEST-CMS user`s manual, Version 3.0. Computational Engineering Mechanics Laboratory, Department of Civil Engineering. State University of New York at Buffalo, Buffalo, NY (1994).

17. B. A. Lerch and J. F. Saltsman, Tensile deformation damage in $\mathrm{SiC}$ reinforced Ti-15V-3Cr-3Al-3Sn. NASA TM-104470 (1991)

18. MSC NASTRAN Version 66, Vols I and II, users manual. The MacNeil Schwendler Corporation (1991).

19. R. T. Bhatt and R. E. Phillips. Laminate behavior for $\mathrm{SiC}$ fiber-reinforced silicon nitride matrix composites. NASA TM-871 54 (1985).

20. H. Bhatt, D. Y. Donaldson, D. P. H. Hasselman and R. T. Bhatt, Role of interfacial carbon layer in the thermal diffusivity conductivity of silicon carbide fiberreinforced reaction-bonded silicon nitride matrix composites. J. Am. cerum. Soc. 2, 334340 (1992)

21. E. Inghels and J. Lamon. An approach to mechanical behavior of $\mathrm{SiC} \mathrm{SiC}$ and $\mathrm{C}$ SiC ceramic matrix composites. Part I, experimental results, J. mater. Sci. 26, 54035410 (1991)

22. R. J Carter. Personal Communication, E. I. DuPont De Nemours, Newark, DF (1992) 
